\title{
Prior hospital admission predicts thirty-day hospital readmission for heart failure patients
}

Donald P. McLaren, Roy Jones, Ronald Plotnik, Wojciech Zareba, Scott McIntosh, Jeffrey Alexis, Leway Chen, Robert Block, Charles J. Lowenstein, Valentina Kutyifa

University of Rochester Medical Center, Rochester, New York, United States

\begin{abstract}
Background: Hospital readmission is a significant health burden. More than $20 \%$ of heart failure (HF) patients are readmitted within 30 days of discharge leading to billions of dollars in health care expenditures. However, the role of prior hospital admissions to predict 30-day readmission for HF patients is not fully understood.

Methods: We retrospectively analyzed HF hospitalization data for 4 years at a single medical center. Association between prior admission and 30-day readmission after HF hospitalization was assessed using a multivariate logistic regression model.

Results: A total of 1,999 patients with index HF hospitalizations were identified, and 366 of them (18\%) were readmitted within 30 days. The rate of readmission was 14\%, 20\%, and $33 \%$ in patients with 0, 1, $\geq 2$ prior admissions. Patients with one prior admission had a $50 \%$ higher risk (confidence interval [CI] 1.10-2.05, $p=0.011$ ) for readmission, while those with $\geq 2$ prior admissions had a more than 3-fold increase in readmission (CI 2.27-4.09, $p<0.001$ ), after adjustments for relevant clinical covariates. Prior hospital admission provided incremental value in predicting readmissions, shown by the significant improvement in the readmission predictive model (C-statistics increased from 0.57 to 0.63). However, neither the length of stay nor recency of prior admission was a significant factor in predicting readmissions.

Conclusions: Hospital admission prior to an index HF hospitalization is associated with a significantly increased risk for 30-day hospital readmission and could be used to identify patients at high-risk for readmission and potentially target interventions to reduce the risk of readmission for these patients. (Cardiol J 2016; 23, 2: 155-162)
\end{abstract}

Key words: heart failure, readmissions, prior admissions, predictive model

\section{Introduction}

Over 5 million Americans suffer from heart failure (HF), and the medical treatment of $\mathrm{HF}$ is associated with significant costs [1,2]. Importantly, $24 \%$ of the patients discharged with a primary diagnosis of $\mathrm{HF}$ are readmitted to the hospital within 30 days, representing a significant public health burden [3-5].

There have been efforts made to predict 30-day hospital readmission by the Centers for Medicare and Medicaid Services [3] and other studies [3, 6-9], some of which include data on prior hospital admissions. However, most of these

Address for correspondence: Valentina Kutyifa, MD, PhD, Heart Research Follow-up Program, Cardiology Division, University of Rochester Medical Center, 265 Crittenden Blvd., PO Box 653, Rochester, NY 14642, USA, tel: 585-275-2676, fax: 585-273-5283, e-mail: Valentina.Kutyifa@heart.rochester.edu 
studies were conducted with Medicare patients, and the predictive accuracy of such models may be suboptimal in other cohorts. Furthermore, it is not known if the length of stay or recency of the prior admission plays a role in predicting readmission rates. Thus, studies regarding the utility of prior admissions to predict 30-day hospital readmission in "all-comers", and further exploration of characteristics of prior admission in predicting readmission are warranted.

In the present analysis, we aimed 1) to evaluate whether prior hospital admissions predict 30-day hospital readmission after an index admission for HF in a real-life cohort, 2) to assess whether the length of stay during prior admission or recency of prior admission plays a role in readmission, and 3) to identify whether information on prior hospital admissions improves the prediction model for 30-day hospital readmission.

\section{Methods}

\section{Study population}

This is a retrospective analysis of hospital admission data in HF patients with an index hospitalization for HF, using data for Strong Memorial Hospital from the Office of Clinical Practice Evaluation at the University of Rochester Medical Center, Rochester, NY, USA.

Patients discharged from the hospital with a primary diagnosis of HF were identified (ICD-9 codes starting with 428 ., plus 402.01, 402.11, 402.91, 404.01, 404.03, 404.11, 404.13, 404.91, 404.93) between January 1, 2007 and June 30, 2011. Hospitalizations of individuals under the age of 18 were excluded from this analysis. Records on hospital admissions between January 1, 2007 and December 31, 2007 were used to derive the number of hospital admissions before January 1, 2008. Hospitalizations with in-hospital death were excluded from the present analysis. In patients with multiple hospitalizations, one index admission was selected at random for analysis, to avoid selection bias.

\section{Definitions and endpoints}

An index admission for each patient was defined as an admission with the primary diagnosis of $\mathrm{HF}$ after January 1, 2008. The variable of interest in this study was the frequency of prior admissions, defined as the number of admissions due to any cause in the 365 days prior to their index admission. We considered any admissions vs. no admission, as well as the effect of 0,1 , and 2 or more admissions, on 30-day hospital readmission rates, as a continuous covariate.

The outcome of our study was 30-day hospital readmission for any cause, defined as a hospitalization for any cause within 30 days after discharge from the index admission for HF.

The study was approved by the University of Rochester Research Subjects Review Board.

\section{Echocardiography data}

Results from 2-dimensional transthoracic echocardiography were obtained during the index admission for $\mathrm{HF}$ when available. Left ventricular (LV) volumes were measured by Simpson's disk method in the apical 4-chamber view and LV ejection fraction (LVEF) was calculated according to the established American Society of Echocardiography protocols [10].

\section{Statistical analysis}

Continuous variables were expressed as mean and standard deviations, categorical variables were summarized as frequencies and percentages. Baseline clinical characteristics were compared between patients with vs. without prior hospital admission in the year before the index admission for HF using the $\chi^{2}$-test for dichotomous variables and the Kruskal-Wallis test for continuous variables, as appropriate.

Multivariate logistic regression analyses were performed to evaluate the risk of 30-day hospital readmissions by prior hospital admissions. Candidate variables in the model were pre-specified during prior literature review, and chosen if they showed significant associations $(\mathrm{p} \leq 0.10)$ with the exposure or the outcome in univariate analyses. There were 29 variables (15 diagnoses) found to have a significant $(\mathrm{p}<0.10)$ association with 30 -day hospital readmission after an index HF admission in the univariate analysis (Supplementary Table 1); these were considered variables for the multivariate logistic regression.

Multivariate logistic regression models were adjusted for relevant clinical covariates selected from the candidate covariates using best subset model regression, forcing age, race, and LVEF in the model. After assessing collinearity among these variables, the model was adjusted for age, race, and LVEF (dichotomized as low LVEF $<40 \%$ vs. relatively preserved $\mathrm{LVEF} \geq 40 \%$ ). We then included prior hospital admissions, the parameter of interest, as a continuous and dichotomized covariate. Covariate effects are reported as odds ratios (OR) and 95\% confidence intervals (CI). We 


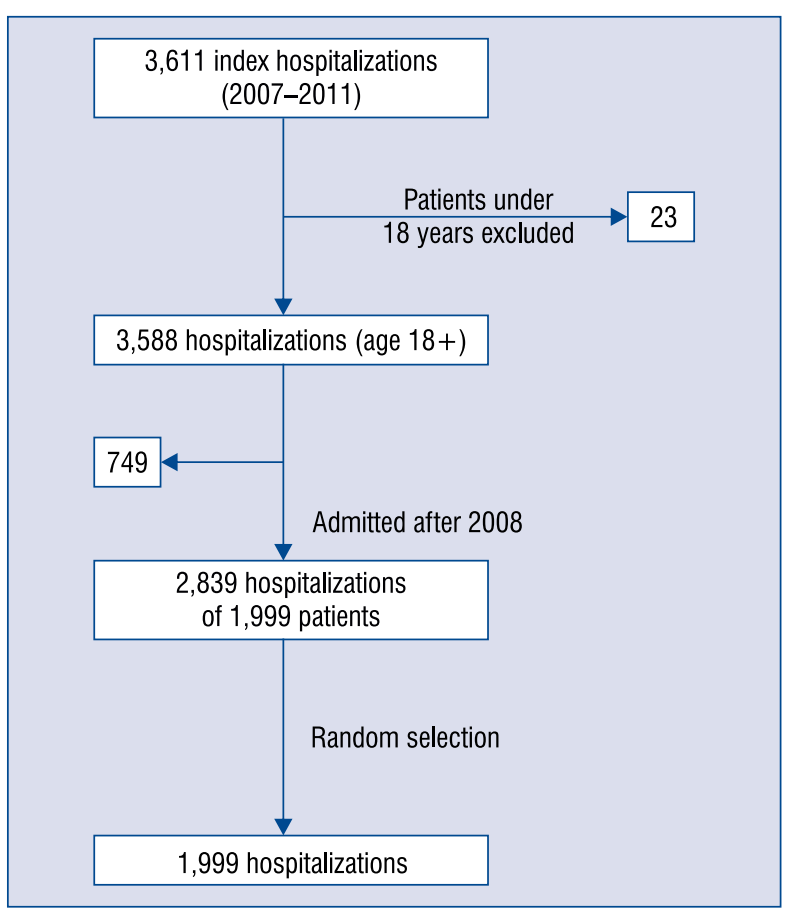

Figure 1. Flowchart of the study population.

reported the overall discriminative value with the receiver-operating characteristic (ROC) curves and results of the $\mathrm{C}$-statistics.

All statistical tests were 2 -sided, and a p-value $<0.05$ was considered statistically significant. All statistical analyses were performed using SAS version 9.3 (SAS, Cary, NC). The statistical analyses were performed by the first author of the manuscript.

\section{Results}

There were a total of 3,611 hospital discharges of patients with the primary diagnosis of HF between January 1, 2007 and June 30, 2011. Twenty-three records were omitted from patients less than 18 years old. In the remaining cohort of 3,588 patients, 749 discharges from the first year were used to calculate prior hospital admission rates for admissions in 2008, as illustrated in Figure 1. Thus, the study population consisted of 1,999 patients with $2,839 \mathrm{HF}$ hospitalizations.

\section{Baseline clinical characteristics}

Clinical characteristics of the patients at index $\mathrm{HF}$ admission are shown in Table 1 . The mean age of the study population was 68 years. A total of 824 (41\%) of the patients were women, and $74 \%$ were white. Common comorbidities were hypertension (36\%), dyslipidemia (42\%), ischemic heart disease
(36\%), and atrial fibrillation (39\%). Patients with prior admission were less often white, more often admitted to the emergency room, and they had more comorbidity, such as atrial fibrillation and other non-cardiovascular diagnoses.

\section{Univariate predictors of 30-day hospital readmission}

There were 366 (18\%) patients with hospital readmission within 30 days after the index HF admission. Patients with higher risk of mortality and severity of illness scores, a greater number of diagnoses, and longer length of stay during their index admission for HF had a significantly higher frequency of 30-day hospital readmission for any cause. Age, gender, marital status, and discharge disposition did not show differences among patients readmitted to the hospital within 30-days vs. those who were not (Supplementary Table 1).

\section{Prior hospital admission predicts 30-day hospital readmission}

The rate of hospital readmission 30 days after an index $\mathrm{HF}$ admission among patients with no prior admission was $14 \%$, compared to $26 \%$ in those with prior admissions $(\mathrm{p}<0.001)$. Figure 2 illustrates the association between the rate of 30-day hospital readmission and the number of hospital admissions in the year prior to the index $\mathrm{HF}$ admission. There were $19.6 \%$ of patients readmitted within 30-days among those with 1 prior admission, $32.3 \%$ among those with 2 prior admissions, and $34.4 \%$ among those with 3 or more prior admission. Because patients with 2 prior admissions and 3 or more prior admissions had similar rates of 30-day readmission, we combined these two groups.

Our results were confirmed in the multivariate logistic regression model, patients with any prior admission had a $31 \%$ higher risk $(\mathrm{p}<0.001)$ of 30-day hospital readmission following an index HF admission (Table 2). When we assessed the risk of 30-day hospital readmission by the number of prior admissions, we found that patients with 1 prior admission had a $47 \%$ increased odds of readmission within 30-days ( $\mathrm{p}=0.015)$; while those with 2 or more prior admissions showed an almost 3-fold increased likelihood of 30-day readmission compared to those with no prior admissions ( $p<0.001$; Table 3 ). Compared to other predictors, prior admissions had the highest $\chi^{2}$ values, thus, associated with the best predictive value in our models.

The ROC curves showed improvement in the $\mathrm{C}$-statistics from 0.57 to 0.62 including prior 
Table 1. Clinical characteristics of the study population at the time of index admission, and stratified by prior hospital admissions.

\begin{tabular}{|c|c|c|c|c|}
\hline Parameters & $\begin{array}{c}\text { All } \\
\text { patients }\end{array}$ & $\begin{array}{l}\text { Patients with } \\
\text { prior hospital } \\
\text { admission (A) }\end{array}$ & $\begin{array}{l}\text { Patients without } \\
\text { prior hospital } \\
\text { admission (B) }\end{array}$ & $\begin{array}{c}\text { P-value } \\
\text { between } \\
A \text { and } B\end{array}$ \\
\hline Number of patients & 1,999 & 700 & 1,299 & \\
\hline \multicolumn{5}{|l|}{ Demographics } \\
\hline Age [years] & $68.2 \pm 15.6$ & $68.2 \pm 15.4$ & $68.2 \pm 15.8$ & 0.916 \\
\hline Female gender & $824(41)$ & $307(44)$ & $517(40)$ & 0.079 \\
\hline White race & $1,485(74)$ & $487(70)$ & $998(77)$ & $<0.001$ \\
\hline Married & $920(46)$ & $313(45)$ & $607(47)$ & 0.388 \\
\hline \multicolumn{5}{|l|}{ Baseline risk scores } \\
\hline Admission type (emergency) & $1,720(86)$ & $628(90)$ & $1,092(84)$ & $<0.001$ \\
\hline Risk of mortality* (major or extreme) & $950(48)$ & $344(49)$ & $606(47)$ & 0.316 \\
\hline Severity of illness** (major or extreme) & $1,366(68)$ & $478(68)$ & $888(69)$ & 0.896 \\
\hline \multicolumn{5}{|c|}{ Characteristics of index heart failure admission } \\
\hline Number of diagnoses on admission & $15.8 \pm 6.1$ & $17.2 \pm 5.9$ & $15.1 \pm 6.1$ & $<0.001$ \\
\hline Length of stay [days] & $7.9 \pm 15.2$ & $8.8 \pm 18.2$ & $7.5 \pm 13.2$ & 0.051 \\
\hline Disposition to home & $1,706(85)$ & $576(82)$ & $1,130(87)$ & 0.005 \\
\hline Readmission in 30-days & $366(18)$ & $181(26)$ & $185(14)$ & $<0.001$ \\
\hline \multicolumn{5}{|c|}{ Co-morbidities at index heart failure admission } \\
\hline Hypertension & $714(36)$ & $219(31)$ & $495(38)$ & 0.002 \\
\hline Hyperlipidemia & $847(42)$ & $301(43)$ & $546(42)$ & 0.676 \\
\hline Ischemic heart disease & $718(36)$ & $147(21)$ & $270(21)$ & 0.910 \\
\hline Left bundle branch block & $122(6)$ & $28(4)$ & $94(7)$ & 0.004 \\
\hline Implantable cardioverter defibrillator & $332(17)$ & $141(20)$ & $191(15)$ & 0.002 \\
\hline Atrial fibrillation & $784(39)$ & $303(43)$ & $481(37)$ & 0.006 \\
\hline \multicolumn{5}{|l|}{ Non-cardiovascular diagnoses } \\
\hline Hyperkalemia & $149(7)$ & $64(9)$ & $85(7)$ & 0.035 \\
\hline End stage renal disease & $136(7)$ & $77(11)$ & $59(5)$ & $<0.001$ \\
\hline Acute respiratory failure & $120(6)$ & $51(7)$ & $69(5)$ & 0.076 \\
\hline Encounter of palliative care & $27(1)$ & $16(2)$ & $11(1)$ & 0.008 \\
\hline Hypertensive chronic kidney disease & $130(7)$ & $64(10)$ & $66(5)$ & $<0.001$ \\
\hline Renal dialysis & $86(4)$ & $44(6)$ & $42(3)$ & 0.001 \\
\hline On supplemental oxygen & $149(7)$ & $74(11)$ & $75(6)$ & $<0.001$ \\
\hline Pleural effusion & $93(5)$ & $49(7)$ & $44(3)$ & $<0.001$ \\
\hline
\end{tabular}

Results are shown as mean \pm standard deviation or numbers and percentages.

*Risk of mortality is the likelihood of dying determined at time of admission, scored as one of four categories: minor, moderate, major, and extreme. **Severity of illness is the extent of physiologic decompensation or organ system loss of function determined at time of admission, scored as one of four categories: minor, moderate, major, and extreme.

admission as a categorical variable, and to 0.63 including prior admission as a continuous variable, as illustrated in Figures 3, 4.

When we assessed the length of stay and the recency of prior hospital admission to the index $\mathrm{HF}$ admission, we did not find a significant association with 30-day hospital readmission rates or further improvement in the fit of the predictive model (data not shown).

\section{Discussion}

We found that hospital admissions in the prior year before an index $\mathrm{HF}$ admission predicted 30-day hospital readmission in a real life patient cohort. The rate of 30-day hospital readmission increased with increasing numbers of prior hospital admissions. Data on prior hospital admission yielded an improvement in the accuracy of the 30-day 


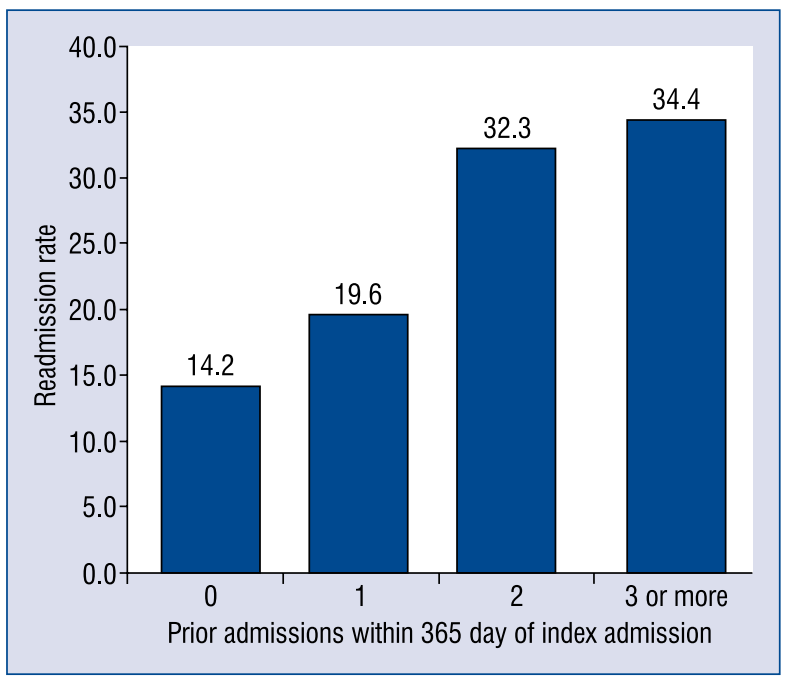

Figure 2. The percentage of patients with 30-day hospital readmission by prior admissions.

hospital readmission predictive model. However, the length of stay or recency of prior admission did not play a role in predicting readmission. Our findings have important clinical implications in identifying a high-risk group for 30-day hospital readmission that could be potentially targeted for interventions to reduce readmissions.

We found a decreased likelihood of 30-day hospital readmission among those with LVEF $<40 \%$, that is consistent with another recent study by Hummel et al. [11]. The possible explanation of this finding is the relatively narrow range of treatment options for HF patients with a preserved ejection fraction (LVEF $>40 \%$ ) compared to those with a reduced ejection fraction $(\mathrm{LVEF}<40 \%)$ [12].

Deriving and validating readmission models that are specific to hospital systems is beneficial for hospitals with focused strategies to reduce readmissions [13]. This is especially important since prior studies focused predominantly on Medicare patients that may not reflect local practices with other cohorts.

The discrimination of our readmission model is comparable to previously published 30-day hospital readmission models. Even though there have been previous studies to predict 30-day hospital readmission in HF patients, only one of these studies utilized prior admissions in their model [7].

Table 2. The effect of the number of prior hospital admission as a continuous covariate on 30-day hospital readmission in a multivariate logistic regression model including significant predictive factors.

\begin{tabular}{lcccc}
\hline \multirow{2}{*}{ Effect } & \multicolumn{2}{c}{ Odds ratio estimates from logistic regression } & \multirow{2}{*}{ P } \\
\cline { 2 - 4 } & Point estimate & $95 \%$ Wald confidence limits & 0.045 \\
\hline $\begin{array}{l}\text { Preserved left ventricular } \\
\text { ejection fraction } \geq 40 \%\end{array}$ & 1.28 & 1.01 & 1.64 & 0.011 \\
$\begin{array}{l}\text { Nonhypertensive } \\
\text { Number of prior hospital }\end{array}$ & 1.40 & 1.08 & 1.82 & $<0.001$ \\
admission in last 365 days & 1.31 & 1.21 & 1.42 & \\
\hline
\end{tabular}

The model is further adjusted for age and race (forced in the model).

Table 3. The effect of prior hospital admission as a categorical covariate on 30-day hospital readmission in a multivariate logistic regression model including significant predictive factors.

\begin{tabular}{|c|c|c|c|c|}
\hline \multirow[t]{2}{*}{ Effect } & \multicolumn{3}{|c|}{ Odds ratio estimates from logistic regression } & \multirow[t]{2}{*}{$\mathbf{P}$} \\
\hline & Point estimate & $95 \% \mathrm{~W}$ & limits & \\
\hline $\begin{array}{l}\text { Preserved left ventricular } \\
\text { ejection fraction } \geq 40 \%\end{array}$ & 1.28 & 1.01 & 1.64 & 0.045 \\
\hline Non-hypertensive & 1.40 & 1.08 & 1.82 & 0.011 \\
\hline $\begin{array}{l}\text { No prior hospital admission } \\
\text { in last } 365 \text { days }\end{array}$ & 1.00 & & & \\
\hline $\begin{array}{l}\text { One prior hospital admission } \\
\text { in last } 365 \text { days }\end{array}$ & 1.47 & 1.08 & 1.99 & 0.015 \\
\hline $\begin{array}{l}\text { Two or more prior hospital } \\
\text { admissions in last } 365 \text { days }\end{array}$ & 2.93 & 2.19 & 3.92 & $<0.0001$ \\
\hline
\end{tabular}

The model is further adjusted for age and race (forced in the model). 


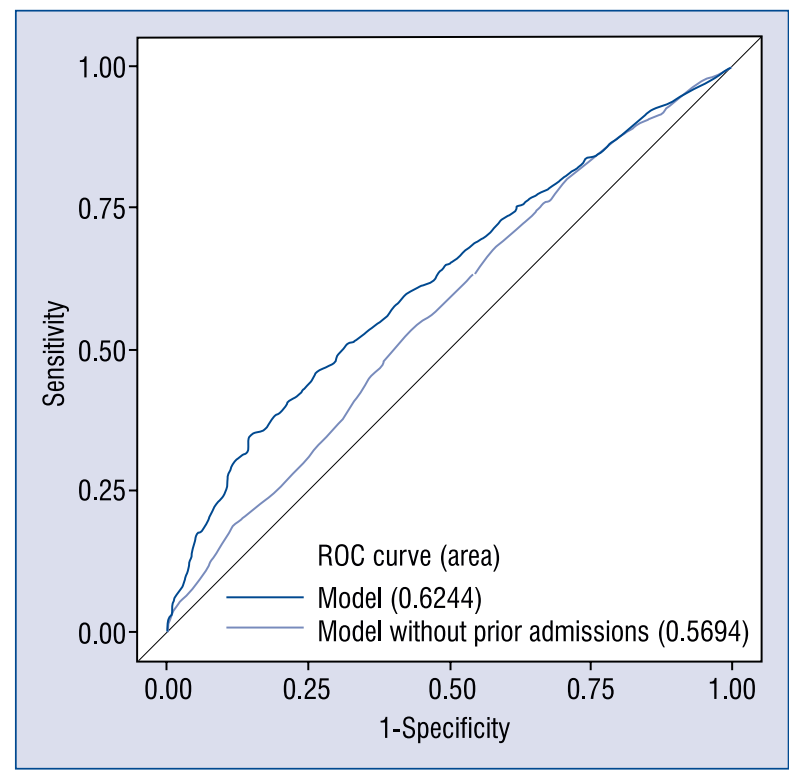

Figure 3. Receiver-operating characteristic (ROC) comparison curves for readmission model to predict 30-day hospital readmissions with and without prior hospital admission modeled as any prior admissions.

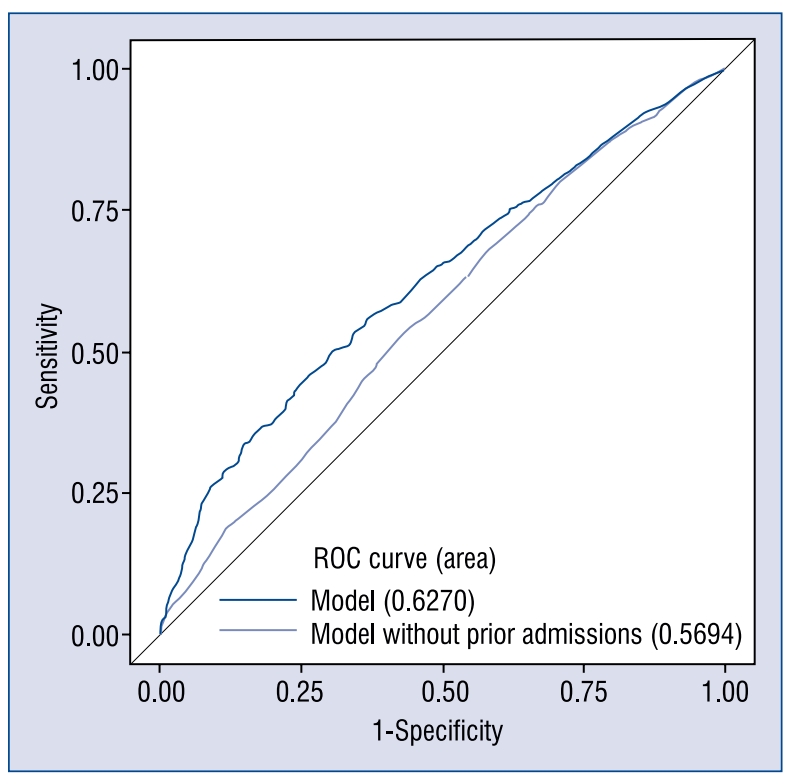

Figure 4. Receiver-operating characteristic (ROC) comparison curves for readmission model using baseline clinical covariates to predict 30-day hospital readmissions with and without prior hospital admission modeled as prior admissions (0, 1, 2 or more).

However, the study by Hummel et al. [7] analyzed only Medicare patients, and therefore the external validity of their findings is limited. In the current analyses, we have demonstrated the importance of prior admissions to predict 30-day hospital readmission in "all-comers", both Medicare and non-Medicare patients.

Similar to a retrospective analysis by Chin and Goldman [14], our study confirmed that the diagnosis of hypertension was protective of readmission. While their study and ours share similarities and focus on a single hospital system, our analysis encompasses a significantly larger patient cohort.

Another study by Eapen et al. [8] derived and validated a logistic regression model on 10 independent predictors of readmission in a large cohort of patients from the Get with the Guidelines Heart Failure Registry. They found a similar predictive accuracy of their model, with a C-statistics of 0.59 . Unlike our study, however, the authors did not include prior admissions in their analysis.

Our study did not address the effect of outpatient follow up on readmission. In a recent report, Casalino et al. [15] found that smaller primary care-based practices had lower rates of potentially preventable hospital admissions compared to larger practices. Outpatient follow-up and physician practice size may have had an effect on the rate of readmission in our population, but our study was not designed to measure this effect. Future studies may benefit from identifying how this factor relates to prior admissions and other health outcomes.

Hummel et al. [7] utilized data from the GAP-HF (Guidelines Applied in Practice-Heart Failure) study and showed that the use of prior admissions improved the discrimination (C-statistics of 0.71 ) of the 30-day readmission prediction model in Medicare patients with HF. Although the authors were able to demonstrate an improvement in an established model utilizing prior admissions, their study included a patient cohort from an earlier time. Management and treatment of HF has significantly improved since then, limiting the validity of their observations. Our study, however, utilized a more current patient population, which may reflect the advances in management of patients with $\mathrm{HF}$ over the last 10 years.

\section{Limitations of the study}

Our study has potential limitations. First, the lack of follow-up data for deaths within 30-days could affect the estimates for 30-day hospital readmission after discharge from the hospital. To determine the impact of this bias on our estimates, we performed a sensitivity analysis utilizing the first admission for each patient in the dataset rather than one at random, confirming similar results to 
the initial models. In addition, there may have been patients who were readmitted to another hospital system, and they may have been erroneously grouped in the no 30-day readmission group.

\section{Conclusions}

Our study shows that hospital admission within the year prior to an index admission for $\mathrm{HF}$ significantly increases the risk of 30-day hospital readmission due to any cause. The rate of 30-day hospital readmission increased with a higher number of prior admissions. Our findings may help clinicians identify patients at high-risk for 30-day hospital readmission following an index HF admission to optimize care and enhance follow-up to improve outcomes.

\section{Acknowledgments}

The authors would like to acknowledge the Heart Failure Care Management Team, an initiative that is currently underway at the University of Rochester Medical Center to prevent heart failure readmissions lead by Dr. Charles Lowenstein.

We would like to acknowledge the contribution of Dr. Karl Schwarz providing data on echocardiography parameters, and Kelly Thevenet-Morrison from the University of Rochester, Rochester, NY for the assistance with the statistical analyses.

Funding source: Dr. McLaren was supported by a grant from the National Institutes of Health (grant no. NIH/NHLBI 5T32HL07937-15, PI Lowenstein).

\section{Conflict of interest: None declared}

\section{References}

1. Go AS, Mozaffarian D, Roger VL et al. Heart disease and stroke statistics: 2014 Update: A report from the American Heart Association. Circulation, 2014; 129: e28-e292. doi: 10.1161/01. cir.0000441139.02102.80.

2. Heidenreich PA, Albert NM, Allen LA et al. Council on Cardiovascular R, Intervention, Council on Clinical C, Council on E, Prevention, Stroke C. Forecasting the impact of heart failure in the United States: A policy statement from the American Heart Association. Circ Heart Fail, 2013; 6: 606-619.

3. Keenan PS, Normand S-LT, Lin $Z$ et al. An administrative claims measure suitable for profiling hospital performance on the basis of 30-day all-cause readmission rates among patients with heart failure. Circulation, 2008; 1: 29-37.

4. Ross JS, Chen J, Lin Z, Bueno H et al. Recent national trends in readmission rates after heart failure hospitalization. Circ Heart Fail, 2010; 3: 97-103.

5. Dharmarajan K, Hsieh AF, Lin $Z$ et al. Diagnoses and timing of 30-day readmissions after hospitalization for heart failure, acute myocardial infarction, or pneumonia. JAMA, 2013; 309: 355-363.

6. Amarasingham $\mathrm{R}$, Patel PC, Toto $\mathrm{K}$ et al. Allocating scarce resources in real-time to reduce heart failure readmissions: A prospective, controlled study. BMJ Qual Saf, 2013; 22: 998-1005.

7. Hummel SL, Katrapati P, Gillespie BW, Defranco AC, Koelling TM. Impact of prior admissions on 30-day readmissions in medicare heart failure inpatients. Mayo Clin Proc, 2014; 89: 623-630.

8. Eapen ZJ, Liang L, Fonarow GC et al. Validated, electronic health record deployable prediction models for assessing patient risk of 30-day rehospitalization and mortality in older heart failure patients. J Am Coll Cardiol Heart Failure, 2013; 1: 245-251.

9. Au AG, McAlister FA, Bakal JA, Ezekowitz J, Kaul P, van Walraven $\mathrm{C}$. Predicting the risk of unplanned readmission or death within 30 days of discharge after a heart failure hospitalization. Am Heart J, 2012; 164: 365-372.

10. Lang RM, Bierig M, Devereux RB et al. Recommendations for chamber quantification: A report from the American Society of Echocardiography's Guidelines and Standards Committee and the Chamber Quantification Writing Group, developed in conjunction with the European Association of Echocardiography, a branch of the European Society of Cardiology. J Am Soc Echocardiogr, 2005; 18: 1440-1463.

11. Hummel SL, DeFranco AC, Skorcz S, Montoye CK, Koelling TM. Recommendation of low-salt diet and short-term outcomes in heart failure with preserved systolic function. Am J Med, 2009; 122: 1029-1036.

12. Campbell RT, Jhund PS, Castagno D, Hawkins NM, Petrie MC, McMurray JJV. What have we learned about patients with heart failure and preserved ejection fraction from DIG-PEF, CHARM-Preserved, and I-PRESERVE? J Am Coll Cardiol, 2012; 60: 2349-2356.

13. Bradley EH, Curry L, Horwitz LI et al. Hospital strategies associated with 30-day readmission rates for patients with heart failure. Circ Cardiovasc Qual Outcomes, 2013; 6: 444-450.

14. Chin MH, Goldman L. Correlates of early hospital readmission or death in patients with congestive heart failure. Am J Cardiol, 1997; 79: 1640-1644.

15. Casalino LP, Pesko MF, Ryan AM et al. Small primary care physician practices have low rates of preventable hospital admissions. Health Affairs, 2014; 33: 1680-1688. 
Supplementary Table 1. Clinical characteristics of the total patient population, and stratified by 30 -day hospital readmission.

\begin{tabular}{|c|c|c|c|c|}
\hline Parameters & All patients & $\begin{array}{l}\text { Patients with } \\
\text { 30-day readmission }\end{array}$ & $\begin{array}{l}\text { Patients without } \\
\text { 30-day readmission }\end{array}$ & $\mathbf{P}$ \\
\hline Number of patients & 1,999 & $366(18 \%)$ & $1,633(82 \%)$ & \\
\hline \multicolumn{5}{|l|}{ Demographics } \\
\hline Age [years] & $68.2 \pm 15.6$ & $67.0 \pm 16.6$ & $68.5 \pm 15.4$ & 0.232 \\
\hline Female gender & $824(41)$ & $163(45)$ & $661(40)$ & 0.154 \\
\hline White race & $1,485(74)$ & $259(71)$ & $1,226(75)$ & 0.088 \\
\hline Married & $920(46)$ & $157(43)$ & $763(47)$ & 0.184 \\
\hline \multicolumn{5}{|l|}{ Baseline risk scores } \\
\hline Risk of mortality* (major or extreme) & $950(48)$ & $196(54)$ & $754(46)$ & 0.010 \\
\hline Severity of illness ${ }^{* *}$ (major or extreme) & $1,366(68)$ & $273(75)$ & $1,093(67)$ & 0.004 \\
\hline \multicolumn{4}{|c|}{ Prior hospital admission before index admission } & $<0.001$ \\
\hline 0 prior admissions & $1,299(65)$ & $185(50)$ & $114(68)$ & \\
\hline 1 prior admissions & $382(19)$ & $75(21)$ & $307(19)$ & \\
\hline 2 or more prior admissions & $318(16)$ & $106(29)$ & $212(13)$ & \\
\hline \multicolumn{5}{|l|}{ Characteristics of index admission } \\
\hline Diagnoses on admission & $15.8 \pm 6.1$ & $17.3 \pm 6.3$ & $15.6 \pm 6.0$ & $<0.001$ \\
\hline Admission type emergency & $1,720(86)$ & $333(91)$ & $1,387(85)$ & 0.003 \\
\hline Length of stay in days & $7.9 \pm 15.2$ & $10.1 \pm 18.5$ & $7.4 \pm 14.3$ & $<0.001$ \\
\hline Disposition to home & $1,706(85)$ & $314(86)$ & $1,392(85)$ & 0.788 \\
\hline Left ventricular ejection fraction $>40 \%$ & $895(48)$ & $181(53)$ & $714(47)$ & 0.046 \\
\hline \multicolumn{5}{|l|}{ Co-morbidities at index admission } \\
\hline Hypertension & $714(36)$ & $109(30)$ & $605(37)$ & 0.009 \\
\hline Dyslipidemia & $847(42)$ & $147(40)$ & $700(43)$ & 0.344 \\
\hline Ischemic cardiomyopathy & $718(36)$ & $126(34)$ & $592(36)$ & 0.510 \\
\hline Left bundle branch block & $122(6)$ & $16(4)$ & $106(7)$ & 0.126 \\
\hline Implantable cardioverter defibrillator & $332(17)$ & $69(19)$ & $263(16)$ & 0.202 \\
\hline Atrial fibrillation & 784 (39) & $153(42)$ & $631(39)$ & 0.263 \\
\hline \multicolumn{5}{|l|}{ Non-cardiovascular diagnoses } \\
\hline Hyperkalemia & $149(7)$ & $42(12)$ & $107(7)$ & 0.001 \\
\hline End stage renal disease & $136(7)$ & $39(11)$ & $97(6)$ & 0.001 \\
\hline Hyposmolality and or hyponatremia & $145(7)$ & $36(10)$ & $109(7)$ & 0.035 \\
\hline Acute respiratory failure & $120(6)$ & $21(6)$ & $99(6)$ & 0.813 \\
\hline Hypertensive chronic kidney disease & $130(7)$ & $39(11)$ & $91(6)$ & $<0.001$ \\
\hline Urinary tract Infection & $209(10)$ & $49(13)$ & $160(10)$ & 0.043 \\
\hline Depressive disorder & $252(13)$ & $52(14)$ & $200(12)$ & 0.307 \\
\hline Family history of diabetes mellitus & $217(11)$ & $53(15)$ & $164(10)$ & 0.014 \\
\hline Hypokalemia & $247(12)$ & $49(13)$ & $198(12)$ & 0.507 \\
\hline Renal dialysis & $86(4)$ & $28(8)$ & $58(4)$ & $<0.001$ \\
\hline On supplemental oxygen & $149(7)$ & $38(10)$ & $111(7)$ & 0.018 \\
\hline Pleural effusion & $93(5)$ & $26(7)$ & $67(4)$ & 0.014 \\
\hline Acute kidney failure & $373(19)$ & $79(22)$ & $294(18)$ & 0.112 \\
\hline Diverticulitis with hemorrhage & $182(9)$ & $42(11)$ & $140(9)$ & 0.081 \\
\hline Hypoxemia & $220(11)$ & $53(14)$ & $167(10)$ & 0.019 \\
\hline
\end{tabular}

Results are shown as mean \pm standard deviation or numbers and percentages.

*Risk of mortality is the likelihood of dying determined at time of admission, scored as one of four categories: minor, moderate, major, and extreme. ${ }^{*}$ Severity of illness is the extent of physiologic decompensation or organ system loss of function determined at time of admission, scored as one of four categories: minor, moderate, major, and extreme. 